\title{
Lack of compliance: a challenge for digital dermoscopy follow-up*
}

\author{
Guilherme Augusto Gadens ${ }^{1}$ \\ DOI: http://dx.doi.org/10.1590/abd1806-4841.20142547
}

Abstract: BACKGROUND: Digital dermoscopy is the gold standard follow-up method for patients with high risk for developing cutaneous malignant melanoma. By comparing the same lesion at different moments, it allows early detection of subtle changes that could suggest the diagnosis of melanoma. Thus, it is clear that the test must be repeated after a period of time, according to time intervals determined by the evaluator.

OBJECTIVES: To evaluate adherence of patients to follow-up examinations using digital dermoscopy.

METHOD: Retrospective analysis of 36 patients who underwent digital dermoscopic examination and total-body photography in a private medical center between September 2010 and January 2013. Results: Only 25\% of the patients returned for followup evaluations.

CONCLUSIONS: Low adherence to digital dermoscopy follow-up could compromise the efficacy of this valuable method. This lack of adherence represents a challenge for the evaluator.

Keywords: Dermoscopy; Dysplastic nevus syndrome; Lost to follow-up; Melanoma; Nevi and melanomas; Patient compliance

\section{INTRODUCTION}

Digital dermoscopic monitoring has proven the most effective method for early detection of melanomas. ${ }^{1}$ The reason of its effectiveness is the fact that this method makes it possible to record an image that will be compared with other images to be collected in follow-up tests. Thus, the most subtle changes in a lesion can be detected and evaluated. Such careful follow-up cannot be achieved using optical dermoscopy because there is no image recording and it relies solely on the evaluator's memory. ${ }^{2}$

Digital dermoscopy, combined with total-body mapping techniques, is particularly recommended in the follow-up of patients at high risk of developing melanomas. In these patients, the assessment of melanocytic lesions at different times is essential for proper follow-up. ${ }^{3}$ Therefore, it is essential that patients undergo follow-up examinations according to medical guidance and respecting the suggested intervals so that the highest effectiveness of the test can be achieved. Poor adherence to follow-up compromises the main advantages of digital dermoscopic monitoring compared to conventional optical dermoscopy, which is performed at a single moment during a medical visit.

\section{OBJECTIVE}

The objective of the present study was to evaluate the rate of adherence to follow-up examinations among patients who underwent digital dermoscopy in our private medical center.

\section{MATERIALS AND METHODS}

Retrospective review of medical records of patients who underwent total-body mapping and digital dermoscopy (using Fotofinder ${ }^{\circledR}$, Fotofinder systems, Inc.) at a private medical center located in Curitiba (PR), Brazil, between September 2010 and January 2013. We included in the study all patients who, after completing the initial examination, were referred to a new follow-up examination to be held before January 2013. We excluded from the study those patients who, despite undergoing the initial assessment, were discharged or referred to perform the next examination after January 2013. Thus, all cases included in the study should have undergone one or more follow-up tests during the study period.

Patients' age was defined as their age at the time of the initial examination. The term "multiple nevi" was used for patients who had 30 or more melanocytic lesions, because these are patients whose follow-up (and subsequent detection of lesions) is difficult to perform without using total body mapping. During the follow-up evaluation, we investigated whether or not the patient returned for the next examination and if the histopathological examination was provided in those cases with indication for excision of any lesions. All patients with indication for removal of any lesions, received written instructions to provide the results of this test.

\footnotetext{
Received on 17.02.13.

Approved by the Advisory Board and accepted for publication on 05.05.2013

* Study conducted at the Instituto de Dermatologia de Curitiba (Inderm) - Curitiba (PR), Brazil.

Financial support: none.

Conflict of interests: none.

Santa Casa de Misericórdia de Curitiba - Curitiba (PR), Brazil.
} 


\section{RESULTS}

Thirty-six patients underwent examination between September 2010 and January 2013. Of these, 21 $(58.3 \%)$ were women and $15(41.7 \%)$ were men. The mean age was 39.4 years (ranging from 4 to 76 years). Only $9(25 \%)$ patients underwent the follow-up examination. Female and male patients' rate of adherence to the follow-up examinations was $23.8 \%$ and $26.7 \%$, respectively.

Twenty-three patients were referred to longterm follow-up (12 months), 6 patients were recommended to have medium-term follow-up ( 6 months), and 7 patients should have a short-term follow-up (3 months). The rates of adherence according to the recommended follow-up were as follows: long-term follow-up $26 \%(6 / 23)$, medium-term follow-up $33 \%$ $(2 / 6)$, and short-term follow-up $14.3 \%(1 / 7)$.

In terms of patients' risk factors, 12 had prior personal history of melanoma, 13 had family history of melanoma, 6 had personal history of Clark's nevus (dysplastic), and 1 had family history of Clark's nevus (Table 1). Sixteen patients had indication for excision of lesions. Of these, only 4 (25\%) provided the results of the histopathological examination to the physician responsible for performing the digital dermoscopy.

\section{DISCUSSION}

The low rate $(25 \%)$ of patients who underwent the follow-up tests recommended by the physician responsible for performing the initial evaluation demonstrates the poor adherence of patients to the proposed follow-up. Previous studies have shown quite different adherence rates, ranging from $46 \%$ to $90 \% ., 5$ In our study, we also found that within the group of patients who underwent follow-up, many patients returned for the next examination after much longer periods than the recommended intervals. It is noteworthy that all

TABLE 1: Characteristics of patients

\begin{tabular}{ll}
\hline Risk factor & $\begin{array}{l}\text { Number of } \\
\text { patients }\end{array}$ \\
\hline PH of melanoma & $9(25 \%)$ \\
PH of dysplastic nevus & $5(13.9 \%)$ \\
FH of melanoma & $8(22.2 \%)$ \\
PH and FH of melanoma & $3(8.3 \%)$ \\
PH of dysplastic nevus + & $1(2.8 \%)$ \\
FH of melanoma & $1(2.8 \%)$ \\
FH of melanoma and & \\
dysplastic nevus & $9(25 \%)$ \\
No other risk factor in addition \\
to the presence of multiple nevi
\end{tabular}

PH = personal history; FH = family history. patients received written documents/reports describing the suggested interval between the follow-up examinations, as well as detailed verbal instructions on the advantages of the examination and the importance and necessity of additional tests.

Another alarming fact is that the vast majority of patients studied had significant risk factors for developing melanoma, which was not enough to motivate the appropriate follow-up (Table 1). Of the 12 patients with a personal history of melanoma, only $50 \%$ (6) returned for the next test. Even among the 7 patients who received formal instructions for shortterm follow-up, i.e., patients with suspicious lesions on initial examination, only $1(14.3 \%)$ returned for reassessment. This finding was not consistent with the results previously described by Argenziano et al., who reported significantly higher rates for short-term follow-up (84\%) compared to medium-term (63\%) and long-term $(30 \%)$ periods. ${ }^{6}$

The lack of dermoscopic follow-up represents a major challenge for the evaluator both because it hinders the early diagnosis of melanoma and also because it interferes with the decision-making process regarding the lesion evaluated. Because of the uncertainty of the follow-up, the evaluator often feels forced to use more aggressive management, indicating excisions of lesions that otherwise could be monitored, provided that follow-up was done in a controlled and periodical manner.

Without a more careful analysis, it is impossible to precisely determine the causes of poor adherence, but some possible reasons are: high cost of test, patients' false sense of safety after initial examination, lack of information/guidance, patients' forgetfulness about the need for follow-up, etc. Among the possible factors mentioned above, the cost of the test certainly plays a significant role in our country, because this type of test still is considered a high-cost examination for most people. Such cost can be even higher for those patients with indication of short-term followup. Thus, some strategies can be used to try to minimize the financial impact on patients, such as providing free of charge 3-month follow-up examination (which is done at our medical center) and charging only for medium-term and long-term tests.

The dermoscopic monitoring of melanocytic lesions makes it possible to establish early diagnosis of cutaneous melanomas, often leading to the diagnosis even before typical dermoscopic findings of the disease are detected. ${ }^{1}$ This can be achieved by means of early detection of subtle changes in the lesion assessed. However, not all changes observed in a melanocytic lesion over time are considered significant. In order to understand the importance of each change, first it is necessary to assess the type of follow-up used 
for a certain lesion. Quite briefly, suspicious melanocytic lesions without criteria suggestive of melanoma (which, if present, would indicate need for excision) should be monitored by means of short-term followup within 3-4 months. ${ }^{7.8}$ On the other hand, patients with known risk factors for melanoma, but without dermatoscopically suspicious lesions, can be monitored using long-term follow-ups within 6-12 months. ${ }^{9}$

In short-term follow-ups, any changes in the lesion should be considered suspicious, indicating the need for surgical removal of the lesion for histopathological evaluation. The only exceptions (i.e., changes that are not clinically significant) for this rule are: ${ }^{7}$

- increase or decrease in the number of horny pseudocysts;

- increased or decreased diffuse pigmentation without structural changes;

- symmetrical increase in the size of the nevus with multiple peripheral globules.

In long-term follow-ups, the following changes are not considered significant: ${ }^{9}$

- increased or decreased diffuse pigmentation;

- change in the number or distribution of brownish globules;

- decrease in the number of black dots;

- resolution of inflammatory reaction;

- replacement of parts of the pigment network with light brown diffuse pigmentation.

However, the risk of melanoma is present if the following changes are observed: ${ }^{9}$

- change in shape;

\section{REFERENCES}

1. Kittler H, Guitera P, Riedl E, Avramidis M, Teban L, Fiebiger M, et al. Identification of clinically featureless incipient melanoma using sequential dermoscopy imaging. Arch Dermatol. 2006;142:1113-9.

2. Skvara H, Teban L, Fiebiger M, Binder M, Kittler H. Limitations of dermoscopy in the recognition of melanoma. Arch Dermatol. 2005;141:155-60.

3. Rezze GG, Soares de Sá BC, Neves RI. Dermatoscopia: 0 método de análise de padrões. An Bras Dermatol 2006;81:261-8.

4. Schiffner R, Schiffner-Rohe J, Landthaler M, Stolz W. Long-term dermoscopic follow-up of melanocytic naevi: clinical outcome and patient compliance. $\mathrm{Br} \mathrm{J}$ Dermatol. 2003;149:79-86.

5. Bauer J, Blum A, Strohhäcker U, Garbe C. Surveillance of patients at high risk for cutaneous malignant melanoma using digital dermoscopy. $\mathrm{Br} J$ Dermatol. 2005;152:87-92

6. Argenziano G, Mordente I, Ferrara G, Sgambato A, Annese P, Zalaudek I. Dermoscopic monitoring of melanocytic skin lesions: clinical outcome and patient compliance vary according to follow-up protocols. Br J Dermatol. 2008;159:331-6

7. Menzies SW, Gutenev A, Avramidis M, Batrac A, McCarthy WH. Short-term digital surface microscopy monitoring of atypical or changing melanocytic lesions. Arch Dermatol. 2001;137:1583-9.
- asymmetric growth;

- change in color;

- reduction in size;

- occurrence of any structures suggestive of melanoma.

When we achieve a better understanding of the possible changes in a melanocytic lesion and the clinical significance of each of these changes, the importance of digital dermoscopic follow-up becomes more evident.

\section{CONCLUSION}

Monitoring melanocytic lesions using digital dermoscopy can only be effective if the patients adhere to the follow-up examinations. The low adherence rates reported in our medical center demonstrate the need to improve patients' awareness and the guidance provided to them. Thus, the active search of patients who should be repeating their tests is a practice to be encouraged in the medical centers that provide such tests. This active search can be conducted by telephone, email, letters, etc. The role of physicians is also crucial, because they should thoroughly instruct patients about the need for follow-up. This responsibility should be shared between the physician responsible for performing the test and the patient's physician, because these are often different health professionals. Although it was not confirmed in our study, the recommendation of follow-up at shorter intervals is another strategy described for greater adhesion. ${ }^{6}$

Our findings are a cause for great concern especially because the vast majority of patients who undergo such examination are at high risk for developing melanoma.

8. Altamura D, Avramidis M, Menzies SW. Assessment of the optimal interval for and sensivity of short-term sequential digital dermoscopy monitoring for the diagnosis of melanoma. Arch Dermatol. 2008;144:502-6.

9. Kittler $\mathrm{H}$, Pehamberger $\mathrm{H}$, Wolff $\mathrm{K}$, Binder M. Follow-up of melanocytic skin lesions with digital epiluminescence microscopy: Patterns of modifications observed in early melanoma, atypical nevi, and common nevi. J Am Acad Dermatol. 2000;43:467-76.

\footnotetext{
MAILING ADDRESS:

Guilherme Augusto Gadens

Rua São Vicente, 55

80030-470 - Curitiba - PR

Brazil

E-mail: guigadens@yahoo.com.br
}

How to cite this article: Gadens GA. Lack of compliance: a challenge for digital dermoscopy follow-up. An Bras Dermatol. 2014;89(2):242-4. 